\title{
The Experiments and Modelling for the Desiccation Cracking
}

\author{
Sayako Hirobe ${ }^{1, a}$ and Kenji Oguni, b \\ ${ }^{1}$ Department of System Design Engineering, Keio University, Yokohama, 2238522, Japan \\ as.hirobe@keio.jp, ${ }^{b}$ oguni@sd.keio.ac.jp
}

Keywords: Desiccation cracks, Pattern formation, PDS-FEM.

\begin{abstract}
The desiccation cracks can be often observed on dry-out soil surfaces. These cracks have a net-like structure and tessellate the surface of the material into polygonal cells hierarchically. The size and the shape of these cells on the final pattern change systematically depending on the specimen thickness and the constraint conditions. In this paper, firstly, we perform the drying experiments of calcium carbonate slurry. Then, we propose the coupled model of desiccation, deformation, and fracture for the problem of the desiccation cracking and perform the weak coupling analysis of the finite element method and particle discretization scheme finite element method. The results of the numerical analysis show the satisfactory agreement with the experimental observation in terms of the process of the crack pattern formation and the change in size of the cells corresponding to the thickness of the specimen.
\end{abstract}

\section{Introduction}

The desiccation cracks can be often observed on dry-out soil surfaces (e.g., deserts, paddy rice fields). During the desiccation process, the drying shrinkage is non-uniform because of the inhomogeneous distribution of the water content. This non-uniform drying shrinkage could result in the generation of the stress and the initiation of the fracture.

The desiccation cracks have a net-like structure and tessellate the surface of the material into polygonal cells hierarchically. These hierarchically-tessellated cells have an almost constant size at the end of the desiccation process. The size and the shape of the cells on the final pattern change systematically depending on the specimen thickness and the constraint conditions. These features of the cell topology and the cell formation are searched on various conditions and materials [1,2]. In any cases, while some varieties can be observed in the size, shape, and the distribution of the cells, the basic topology of the cells and the cell formation process (i.e., the net-like structure of the cracks, hierarchical sequence of the cell formation, the systematic change in size and shape of the cells) are persistently conserved.

In spite of the importance of the inhomogeneous water distribution, most of the models proposed in previous researches assume the homogenous water distribution (uniform shrinkage) [3-5]. While this assumption might be sufficient in the case of thin-layer specimen where the gradient of the water distribution can be neglected, it cannot apply for the thick-layer specimen. The other models cannot couple the water movement, deformation, and fracture completely [6,7]. While the assumption of the homogeneous water distribution or the pseudo-coupling analysis is sufficient to reproduce the crack initiation or the final crack pattern in the limited case, the process of the crack pattern formation cannot be reproduced.

In this paper, the coupled model of desiccation, deformation, and fracture is proposed for the problem of the desiccation crack phenomenon. We perform the weak coupling analysis of the finite element method for the desiccation and particle discretization scheme finite element method (PDS-FEM) [8] for the seamless analysis of the deformation and fracture based on this coupled model. The results of the numerical analysis are compared with the results of drying experiments of calcium carbonate slurry and validated qualitatively. 


\section{Laboratory Experiments of Calcium Carbonate Slurry}

We perform the drying experiments of calcium carbonate slurry to observe the crack patterns corresponding to the thickness of the specimen and the process of the crack pattern formation. The calcium carbonate slurry was prepared at water content rate $90 \%$. Then, the slurry was poured into the rectangular acrylic container with a size of $100 \times 100 \times 50 \mathrm{~mm}$. The thickness of the specimen was set as $5 \mathrm{~mm}, 10 \mathrm{~mm}, 20 \mathrm{~mm}$, and $30 \mathrm{~mm}$. The slurry was dried in the air $\left(20{ }^{\circ} \mathrm{C}\right.$ temperature and at $50 \%$ relative humidity) until the entire of the specimen dried out completely. The rate of weight reduction and the change in volumetric water content was measured.

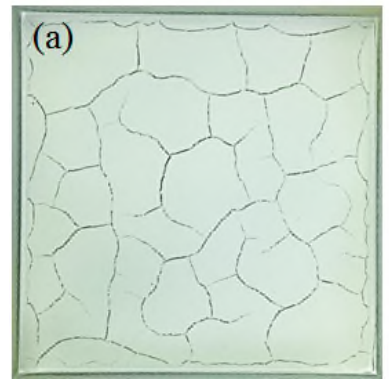

(a) $5 \mathrm{~mm}$

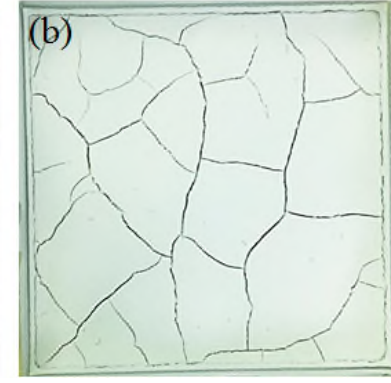

(b) $10 \mathrm{~mm}$

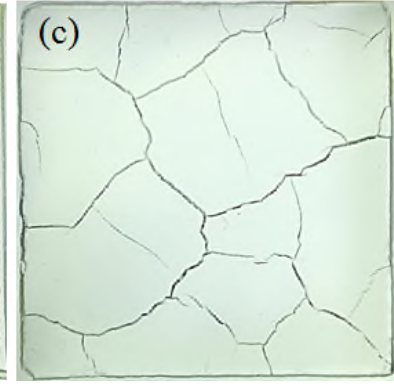

(c) $20 \mathrm{~mm}$

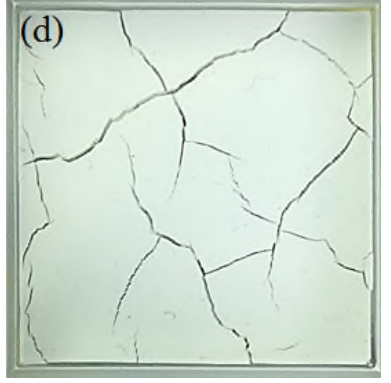

(d) $30 \mathrm{~mm}$

Fig. 1 The surface crack pattern of the drying experiments of calcium carbonate slurry.
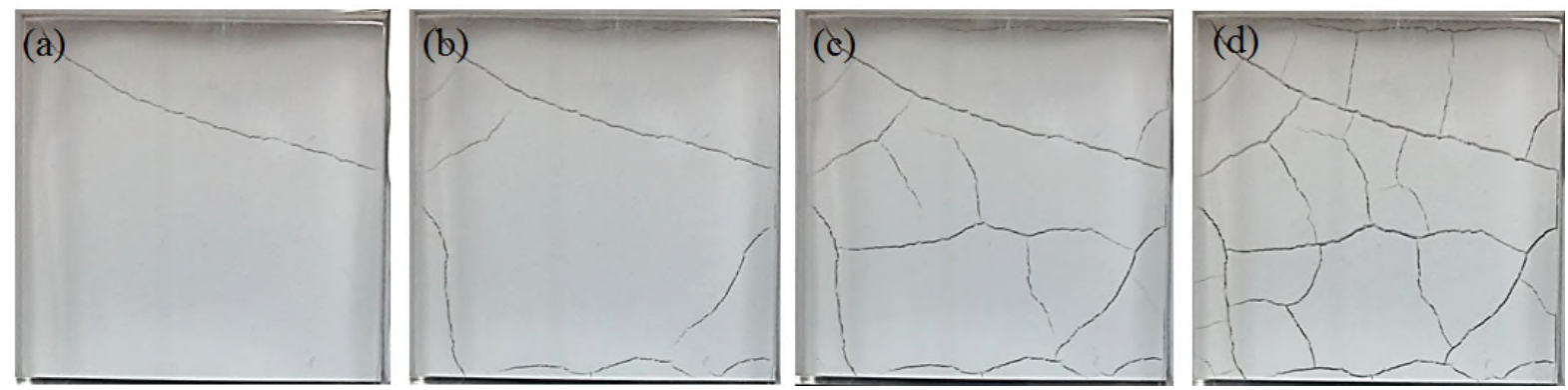

Fig. 2 The crack propagation process observed on the drying experiments in the case of $10 \mathrm{~mm}$ thickness.

The excessive water layer disappeared at the volumetric water content $56.6 \%$ and the crack initiated at the volumetric water content $22.4 \%$. The crack pattern formation terminated at the volumetric water content $20.4 \%$; the crack pattern formation terminated before the entire specimen dries out. The volumetric water content reduces almost linearly during desiccation.

Fig. 1 shows the final crack patterns on the top surface of the specimen with different thickness. The cells framed by the cracks have an almost constant size in each specimen and the averaged size of the cells increase with the increase of the specimen thickness.

The process of the crack pattern formation in the case of $10 \mathrm{~mm}$ thickness specimen is show in Fig. 2. Some long cracks initiates on the edge of the specimen and extends to the other edge traversing the specimen on the initial stage of the desiccation cracking (Fig. 2 (a) and (b)). These cracks do not branch and form the largest structure of the cells. These cracks can be considered as the primary cracks. Then, relatively short cracks appear to tessellate the lager cells (Fig. 2 (c) and (d)). These cracks often branch and terminate when they meet the existing cracks. These cracks can be considered as the secondary cracks. The tessellation by the secondary cracks continues until the termination of the crack propagation.

\section{Modelling of the Desiccation Cracking}

The water movement inside the mixture of the powder and the water is described by the diffusion equation: 


$$
\frac{\partial \theta}{\partial t}=D \nabla^{2} \theta
$$

where $\theta$ is a volumetric water content and $D$ is moisture diffusion coefficient. Here, the gravitational effect is neglected and moisture diffusion coefficient $D$ is assumed as constant in the problem of the desiccation crack phenomenon; the model of the water movement is simplified to the linear diffusion equation. Consider a permeable and linearly elastic body $\Omega$ with external boundary $\Gamma^{1}$. When the water evaporated from the boundary $\Gamma^{1}$, the water distribution in $\Omega$ is given by the next boundary value problem:

$$
\begin{cases}\frac{\partial}{\partial t} \theta(\boldsymbol{x}, t)=D \nabla^{2} \theta(x, t) & \boldsymbol{x} \in \Omega \\ D \frac{\partial}{\partial \boldsymbol{n}} \theta(\boldsymbol{x}, t)=-Q^{1}(\theta) & \boldsymbol{x} \in \Gamma^{1} \\ \theta(\boldsymbol{x}, 0)=\bar{\theta}, & \end{cases}
$$

where $Q^{1}(\theta)$ is a water flux due to the evaporation from the boundary $\Gamma^{1}$. Here, only the liquid water movement is considered.

For the coupling of the desiccation and fracture, the effect of the crack surfaces should be embedded in the water movement. In this research, the crack surface $\Gamma^{2}$ is considered as a newly created evaporation surfaces and a shield for the permeable flow. The evaporation from the crack surface $\Gamma^{2}$ can be expressed by modifying the Neumann boundary condition of Eq. (2):

$$
D \frac{\partial}{\partial \boldsymbol{n}} \theta(\boldsymbol{x}, t)=-\boldsymbol{Q}^{\alpha}(\theta) \quad \boldsymbol{x} \in \Gamma^{\alpha}(\alpha=1,2)
$$

where $Q^{2}(\theta)$ is a water flux from the crack surface $\Gamma^{2}$ due to evaporation.

The shield for the permeable flow can be expressed as the elimination of the permeability in the normal direction to the crack surface $\Gamma^{2}$. The Darcy's low in orthonormal coordinate system $\left\{\boldsymbol{e}_{i}\right\}$ is

$$
\boldsymbol{J}=-D \nabla \theta
$$

where $\boldsymbol{J}$ is a water flux vector expressed in the $\left\{\boldsymbol{e}_{i}\right\}$ coordinate system. We define the orthonormal coordinate system $\left\{\boldsymbol{e}_{i}^{\prime}\right\}$ with $\boldsymbol{e}_{3}^{\prime}$ in the normal direction of the crack surface $\Gamma^{2}$ with the coordinate transform matrix $T_{i j}$ :

$$
T_{i j}=\boldsymbol{e}_{i}^{\prime} \cdot \boldsymbol{e}_{j}
$$

Then, the projection of $\boldsymbol{J}$ on $\Gamma^{2}\left(\right.$ denoted as $\boldsymbol{J}^{c}$ ) is expressed in the $\left\{\boldsymbol{e}_{i}\right\}$ coordinate system as

$$
J_{i}^{c}=T_{j i} P_{j k} T_{k l} J_{l}
$$

where the projection matrix which eliminates the permeable flow in the direction normal to $\Gamma^{2}$ is

$$
P_{i j}= \begin{cases}1 & \text { if } i=j=1,2 \\ 0 & \text { otherwize. }\end{cases}
$$

The introduction of $\boldsymbol{J}^{c}$ in the place of the $\boldsymbol{J}$ is equivalent to introducing the anisotropic moisture diffusion coefficient in Eq. (2). We solve the boundary value problem for the water movement Eq. (2) by the ordinary FEM with linear tetrahedral elements.

For the coupling of desiccation and deformation, the volume shrinkage corresponding to the reduction of the volumetric water content $\theta$ should be embedded in the infinitesimal deformation problem. In the case of the desiccation crack phenomenon, the total strain $\varepsilon_{i j}$ is composed of the elastic strain $\varepsilon_{i j}{ }^{e}$ and the shrinkage strain $\varepsilon_{i j}{ }^{e}$. Since the shrinkage strain $\varepsilon_{i j}{ }^{e}$ does not contribute to the generation of the stress, the stress strain relationship becomes 


$$
\sigma_{i j}=c_{i j k l}\left(\varepsilon_{k l}-\varepsilon_{k l}^{s}\right) \text {. }
$$

Therefore, the strain energy I for the problem of the desiccation cracking is defined as

$$
\mathrm{I}=\int_{\Omega} \frac{1}{2}\left(\varepsilon_{i j}-\varepsilon_{i j}^{s}\right) c_{i j k l}\left(\varepsilon_{k l}-\varepsilon_{k l}^{s}\right) d V .
$$

In this paper, the deformation and fracture are seamlessly treated by PDS-FEM. For the numerical evaluation of the functional I in Eq. (9), PDS-FEM applies the particle discretization for the physical field by using a pair of the conjugate geometries; Voronoi tessellations and Delaunay tessellations. The Delaunay block becomes a tetrahedron on the three-dimensional problem. Then, the discretized strain energy I is

$$
\hat{\mathrm{I}}=\sum_{\beta=1}^{M} \frac{1}{2}\left(\varepsilon_{i j}^{\beta}-\varepsilon_{i j}^{s \beta}\right) c_{i j k l}^{\beta}\left(\varepsilon_{k l}^{\beta}-\varepsilon_{k l}^{s \beta}\right) \Psi^{\beta} .
$$

where $M$ is the number of Delaunay blocks and $\Psi^{\beta}$ is the volume of the $\beta$-th Delaunay blocks. The displacement $u_{i}^{\alpha}$ minimizing the discretized strain energy Eq. (10) is satisfying the next equation of the force equilibrium:

$$
\sum_{\gamma=1}^{N} K_{i k}^{\alpha \gamma} u_{k}^{\gamma}=f_{i}^{\alpha},
$$

where,

$$
\begin{aligned}
& K_{i k}^{\alpha \gamma}=\sum_{\beta=1}^{M} B_{j}^{\beta \alpha} c_{i j k l}^{\beta} B_{l}^{\beta \gamma} \Psi^{\beta}, \\
& f_{k}^{\alpha}=\varepsilon_{i j}^{s \beta}\left(c_{i j k l}^{\beta} B_{l}^{\beta \alpha}\right) \Psi^{\beta} .
\end{aligned}
$$

Here, $N$ is the number of Volonoi blocks, $B_{j}^{\beta \alpha}$ is a six-by-twelve strain-displacement matrix, and $K_{i k}^{\alpha \gamma}$ is a global stiffness matrix. The shrinkage strain $\varepsilon_{i j}{ }^{e}$ can be derived from the volume strain obtained by the reduction of the volumetric water content $\theta$. Thus, the effect of volume shrinkage due to desiccation is embedded in the equation of force equilibrium Eq. (11) as $\varepsilon_{i j}{ }^{s \beta}$ in Eq. (13).

Once the traction on the boundary of Voronoi blocks reach to the tensile strength, the interaction between the Voronoi blocks is lost. This loss of the interaction is introduced by changing a stiffness matrix $K_{i k}^{\alpha \gamma}$ and strain-displacement matrix $B_{j}^{\beta \alpha}$ on the fractured boundary of the Voronoi blocks on PDS-FEM.

\section{Numerical Analysis for the Desiccation Crack Phenomenon}

We perform the numerical analysis to reproduce the crack patterns with different thickness of the specimen and the crack propagation process observed in the laboratory experiments of calcium carbonate slurry. The time history of the distribution of the water content is obtained by the FEM analysis with a constant time step $\Delta t=0.1$ hour. Then, the seamless analysis for the deformation and the fracture is performed by PDS-FEM at each time step (quasi-static analysis). Since the time scale for the desiccation expressing by the diffusion equation and the fracture have a strong contrast, we performed the weak coupling analysis for the desiccation and fracture (i.e., FEM analysis and the analysis of PDS-FEM). To capture the effect of the fracture surfaces on the desiccation and deformation promptly, the time step is reduces to $\Delta t=0.01$ hour when the maximum traction among all elements reached to the $97 \%$ of the tensile strength.

We set model sizes and the boundary conditions to fit the conditions of the drying experiments of calcium carbonate slurry. The width and the height of the analysis model were set as $100 \mathrm{~mm}$ and the depth was set as $5 \mathrm{~mm}, 10 \mathrm{~mm}, 20 \mathrm{~mm}$, and $30 \mathrm{~mm}$. The nodal displacement of the sides and the bottom 
surfaces of the analysis model were constrained in all directions; this constraint condition is expressing the adhesion between the slurry and the container wall on the drying experiments. The water evaporates from the only top surface of the analysis model. We prepared the finite element models with unstructured mesh for each models.

The measurable parameters were also determined from the drying experiments of calcium carbonate slurry. The initial volumetric water content is $56.6 \%$; the excessive water layer disappeared in the drying experiments. The analysis was stopped when the volumetric water content reached to the 20.4\%; the crack pattern formation terminated in the drying experiments. The evaporation speed on the top surface $Q^{1}$ is $0.088 \mathrm{~kg} / \mathrm{m}^{2} \cdot$ hour.

The other parameters which are not measured in the drying experiments of calcium carbonate slurry were determined from the drying experiments of clayey silt in previous researches [1,9]. The moisture diffusion coefficient $D$ is $3.6 \times 10^{-2} \mathrm{~m}^{2} /$ hour, Young's modulus is $5.0 \mathrm{MPa}$, and tensile strength is $1.6 \mathrm{MPa}$. The evaporation speed on the crack surfaces $Q^{2}$ was set as $50 \%$ of the evaporation speed on the top surface because the opening width of the cracks is narrow.

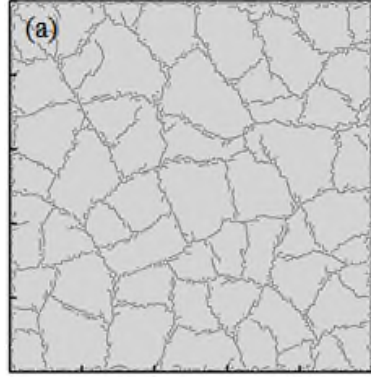

(a) $5 \mathrm{~mm}$

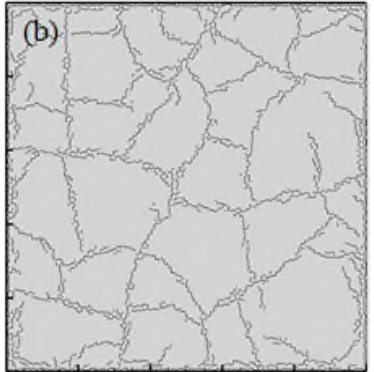

(b) $10 \mathrm{~mm}$

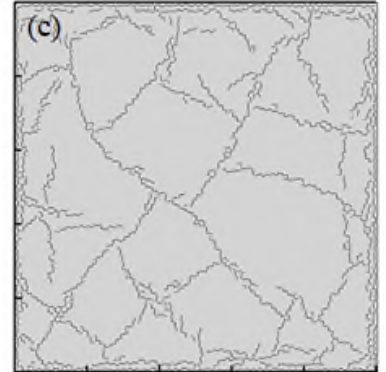

(c) $20 \mathrm{~mm}$

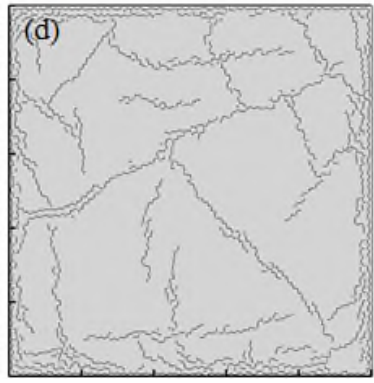

(d) $30 \mathrm{~mm}$

Fig. 3 The final crack pattern on the top surface reproduced by the numerical analysis.
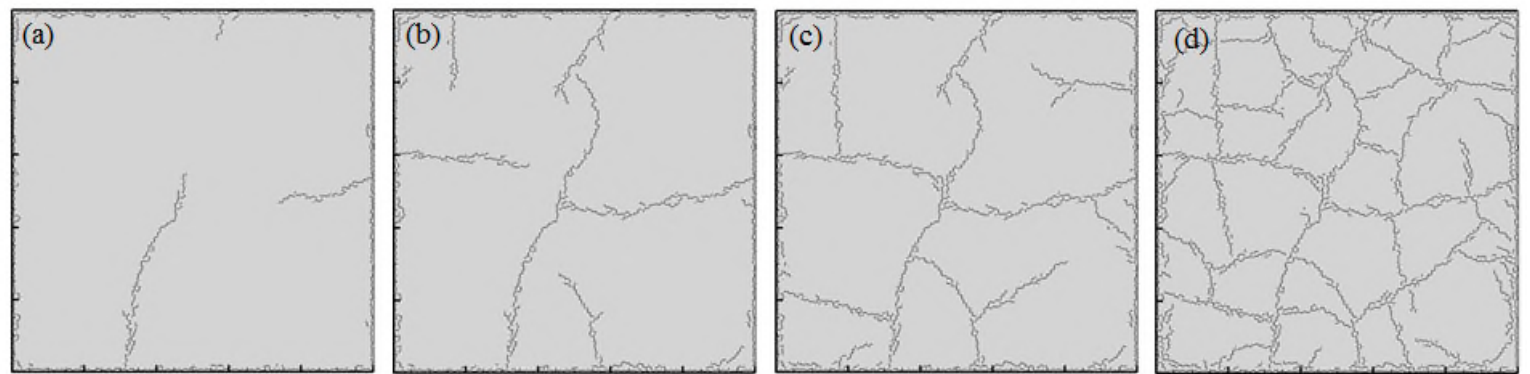

Fig. 4 The crack propagation process on te numerical analysis in the case of 10mm thickness.

Fig. 3 shows that crack pattern appearing on the top surface of the analysis models with different thickness. The cracks have net-like appearance and form polygonal cells. The size of the cells is almost constant on each thickness and the averaged cell size increases with the increase of the thickness. These features of the crack patterns and the increase tendency of the averaged cell size coincide with the observation of the drying experiments of calcium carbonate slurry.

Fig. 4 shows the crack propagation process in the case of $10 \mathrm{~mm}$ thickness. Some long cracks initiate on the edge of the top surface and extend traversing the top surface (Fig. 4 (a) and (b)). These cracks can be considered as primary cracks appearing on the initial stage of the desiccation cracking. Then, relatively short cracks propagate to tessellate the lager cells (Fig. 4 (c) and (d)). These cracks often branch and terminate when they meet other cracks; these features indicate the emergence of the secondary cracks. This hierarchical sequence of the cell formation can be also observed in the drying experiments. 


\section{Conclusion}

In this paper, the coupling model of desiccation, deformation, and fracture are proposed and validated by comparing the result of the numerical analysis and the drying experiments of calcium carbonate slurry. We perform the weak coupling analysis of FEM analysis and the analysis of PDS-FEM. The numerical analysis can reproduce particular pattern of cracks (have net-like structure and form polygonal cells). The increase tendency of the averaged cell size depending on the specimen thickness and the hierarchical sequence of the cell formation are also reproduced by the numerical analysis. These results of the numerical analysis show the satisfactory agreement with the observation of the drying experiments of calcium carbonate slurry. Therefore, we can conclude that the proposed model and the numerical analysis method can capture the fundamental features of the desiccation crack phenomenon. For the quantitative discussion, we need to search the effect of the parameters on the crack patterns.

\section{References}

[1] H. Peron, T. Hueckel, L. Laloui and L. B. Hu, Fundamentals of desiccation cracking of fine-grained soils: experimental characterization and mechanisms identification, Can. Geotech. J. 46 (2009) 1177-1201.

[2] A. Groisman and E. Kaplan, An experimental study of cracking induced by desiccation, Europhys. Lett. 25 (2006) 415-420.

[3] H. J. Vogel, H. Hoffmann, A. Leopold and K. Roth, Studies of crack dynamics in clay soil II. a physically based model for crack formation, Geoderma. 125 (2005) 213-223.

[4] J. Sima, M. Jiang and C. Zhou, Numerical simulation of desiccation cracking in thin clay layer using 3D discrete element modelling, Comput. Geotech. 56 (2014) 168-180.

[5] R. Rodríguez, M. Sánchez, A. Ledesman and A. L loret, Experimental and numerical analysis of desiccation of mining waste, Can. Geotech. J. 44 (2007) 644-658.

[6] H. Peron, J. Y. Delenne, L. Laloui and M. S. El Youssoufi, Discrete element modelling of drying shrinkage and cracking of soils, Comput. Geotech. 36 (2008) 61-69.

[7] G. Musielak and T. Śliwa, Fractureing of clay during drying: modelling and numerical simulation, Transport. Porous. Med. 95 (2012) 465-481.

[8] K. Oguni, M. L. L. Wijerathne, T. Okinaka and M. Hori, Crack propagation analysis using PDSFEM and comparison with fracture experiment, Mech. Mater. 41 (2009) 1242-1252.

[9] H. Nahlawi and J. K. Kodikara, Laboratory experiments on desiccation cracking of thin soil layers, Geotech. Geol. Eng. 24 (2006) 1641-1664. 